\title{
A practical scheduling and power/constellation allocation for three relay networks
}

\author{
Paolo Baracca*, Stefano Tomasin and Nevio Benvenuto
}

\begin{abstract}
Relays are being deployed in both fourth generation cellular systems and wide metropolitan area networks in order to increase coverage and spectral efficiency. In this article, we consider a network with three half-duplex relays assisting the transmission from a source towards a destination. By assuming that transmission time consists of a sequence of phases, we develop a transmission scheme in which spatial multiplexing is achieved by alternating the transmission of a single relay in odd phases and a couple of relays employing Slepian-Wolf encoding in even phases. Moreover, to simplify Slepian-Wolf decoding (consisting of a scheme with successive interference cancelation), we propose a suboptimal scheme in which cooperative relays employ quantized QAM constellations to yield at the destination the desired QAM constellation with no interference. We formalize the problem of maximizing network spectral efficiency optimizing (a) QAM size, (b) power allocation, (c) time allocation, and (d) relays transmitting and receiving at each phase. The performance of the proposed scheme is compared against existing techniques in typical wireless scenarios showing the merits of the proposed approach.
\end{abstract}

Keywords: relay network, cooperative systems, quadrature amplitude modulation

\section{Introduction}

Relays or cooperative networks where the transmission between a source and a destination is assisted by other nodes have shown to be an interesting solution to implement distributed multiple input multiple output (MIMO) systems. Initial works on relay networks have considered a single relay node [1] and various transmission techniques, including decode and forward, amplify and forward, and compress and forward [2-4]. As relay nodes are being deployed, e.g., in forth generation cellular networks and in wide metropolitan area networks [5], there is an increasing interest in using more than one relay. In fact beamforming can be used by cooperative relays with the aim of transmitting coherently towards the destination. Networks with multiple relays have been widely studied under the full-duplex assumption in $[6,7]$. The achievable rates for various techniques have been derived in [8] for the case of half duplex relays in the absence of interference for a network with two relays.

In this article, we consider a network with three relays operating in half duplex mode. In order to limit the

\footnotetext{
* Correspondence: baraccap@dei.unipd.it

Department of Information Engineering, University of Padova, Padova, Italy
}

complexity of the considered scenario, we assume that relays cannot communicate with each other, but only with the source and the destination. Furthermore, we consider that the transmission time is a sequence of phases, where in odd phases a subset of relays is transmitting and the rest is receiving, while in the even phases the role of the relays is swapped. For this scenario we formulate the optimization problem that maximizes the throughput from the source to the destination, considering transmission power constraints at each node. The optimization includes both the choice of the subset of relays transmitting and receiving at each phase and the duration of even and odd phases. Two solutions are proposed. The first is based on the Slepian-Wolf theorem [9] for the transmission of private and common information and requires successive interference cancelation at the destination. In the second solution we adapt the technique developed in [10] for downlink multi-cell scenario by allowing the simultaneous transmission of relays towards the destination in such a way that a single QAM symbol is received, and thus not requiring successive interference cancelation. The performance of the proposed solutions is compared with respect to existing approaches, showing 
a significant improvement of the network throughput in typical wireless scenarios.

The rest of the article is organized as follows. In Section 2, we introduce the system model of the network with three relays and briefly summarize existing approaches for the cooperative transmission. In Section 3 , we propose the new solutions deriving the achievable network throughput. Numerical results are presented and discussed in Section 4. Lastly, conclusions are outlined in Section 5.

\section{System model}

We consider the relay network shown in Figure 1 consisting of three relays assisting a source $S$ transmitting towards a destination $D$. All the nodes are equipped with a single antenna, and we indicate with $h_{i} \in \mathbb{C}, g_{i} \in \mathbb{C}, i=$ $1,2,3$, the flat-fading channel between $S$ and relay $i$ and between relay $i$ and $D$, respectively. $S$ transmits a signal $x$, and the signal received by relay $i$ can be written as

$$
y_{i}=h_{i} x+w_{i}
$$

where $w_{i}$ is the additive white complex Gaussian noise with zero mean. We indicate the signal transmitted by relay $i$ with $z_{i}$ and consider a unitary power constraint for each node in the network, i.e., $\mathrm{E}\left[|x|^{2}\right] \leq 1, \mathrm{E}\left[\left|z_{i}\right|^{2}\right] \leq$ $1, i=1,2,3$. By assuming channel state information of the relay-destination link at the relay, the transmission performed by relays can be done coherently adjusting

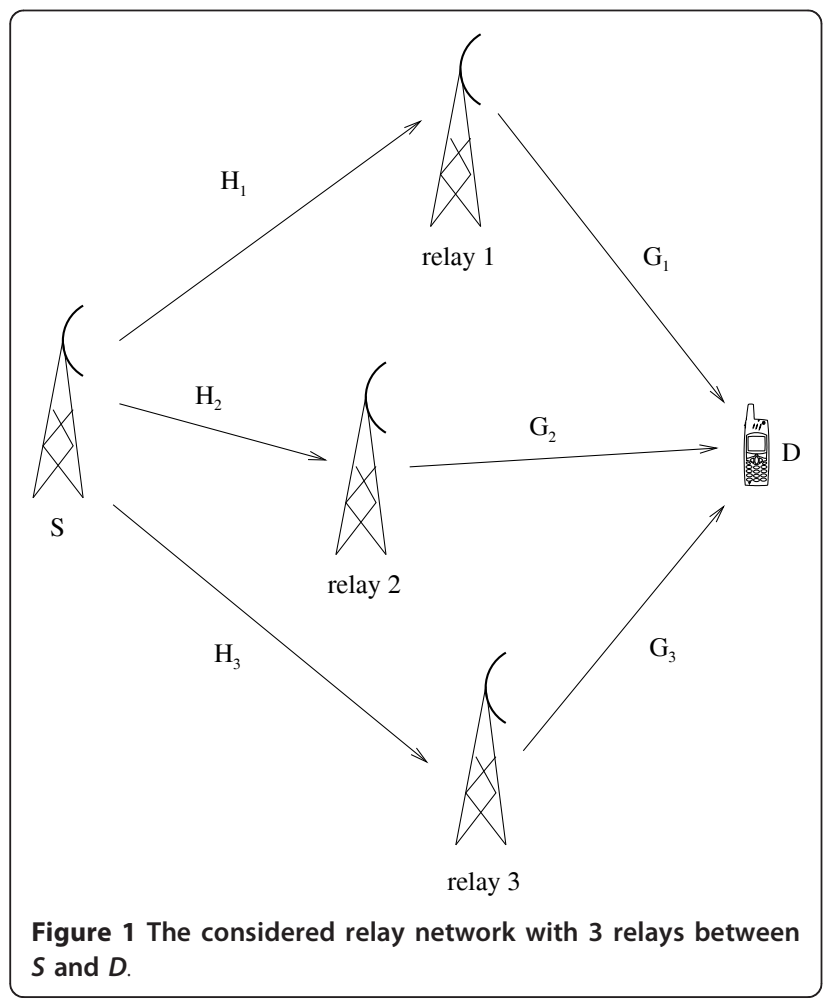

the phases of $z_{i}, i=1,2,3$. For this reason and without loss of generality, in the following we only consider the channel gains

$$
\begin{aligned}
& H_{i}=\left|h_{i}\right|^{2}, \\
& G_{i}=\left|g_{i}\right|^{2} .
\end{aligned}
$$

Without loss of generality, we assume that all noises have unitary variance, while the effective signal to noise ratio (SNR) at the receiver is obtained by a proper scaling of the channel gains, see (19). We assume that a central controller knows all channels, correspondingly allocates resources to nodes, including power, and constellation sizes. Hence, the determined network spectral efficiency can be assumed as a bound for cases where only a partial channel state information is available.

Nodes operate in half-duplex mode, i.e., they cannot transmit and receive simultaneously. Each relay alternates a phase in which it receives data from the source and a phase when it transmits to the destination. No communication among relays is allowed. Moreover, we assume no direct transmission from $S$ to $D$ because of shadowing or the long distance between $S$ and $D$. Let the time used for two consecutive phases be unitary. The odd phases are assigned a time $\lambda$, and the even phases are assigned a time $1-\lambda$, where the parameter $\lambda$ will be optimized. The scheduling of transmission is then fully characterized by the variable

$$
\delta_{i}=\left\{\begin{array}{l}
0, \text { relay } i \text { transmits during even phases, } \\
1, \text { relay } i \text { transmits during odd phases, }
\end{array}\right.
$$

for $i=1,2,3$. We will see that the scheduler must know the channel gains for all relays and all phases in order to compute $\lambda$ and assign $\delta_{i}$.

We first review transmission schemes proposed in the literature where all relays receive in odd phases and transmit in even phases, i.e., $\delta_{i}=0, i=1,2$, 3. In detail, we consider the amplify-and-forward (AF), the decodeand-forward (DF), and the broadcast-multiaccess (BM) techniques. Let us denote the link spectral efficiency for a given SNR $\eta$ as

$$
C(\eta)=\log _{2}(1+\eta) \text {. }
$$

\subsection{Amplify-and-forward}

In AF each relay simply retransmits a scaled version of the received signal, i.e., $z_{i}=\gamma_{i} y_{i}$, observing the unitary power constraint $\gamma_{i}^{2}\left(H_{i}+1\right) \leq 1$. The signal received at node $D$ can be written as

$$
r=\sum_{i=1}^{3} \sqrt{G_{i}} z_{i}+w_{D}=x \sum_{i=1}^{3} \gamma_{i} \sqrt{G_{i} H_{i}}+w_{D}+\sum_{i=1}^{3} w_{i} \gamma_{i} \sqrt{G_{i}},
$$


where $w_{D}$ is complex Gaussian with zero mean and unitary variance. Note that with AF no optimization of the time-allocation $\lambda$ is performed. Indeed, each relay retransmits the whole received signal from $S$ towards $D$, therefore equal-time has to be assigned to both phases, i.e., $\lambda=1 / 2$. The spectral efficiency is obtained by (4), where from (5) the signal power is $\left|\sum_{i=1}^{3} \sqrt{G_{i} H_{i}} \gamma_{i}\right|^{2}$ and the noise power is $1+\sum_{i=1}^{3} G_{i} \gamma_{i}^{2}$. Scaling factors $\gamma_{i}$ are selected in order to maximize the network spectral efficiency, i.e.,

$$
R^{(\mathrm{AF})}=\max _{\gamma_{i} \geq 0} \frac{1}{2} C\left(\frac{\left|\sum_{i=1}^{3} \sqrt{G_{i} H_{i}} \gamma_{i}\right|^{2}}{1+\sum_{i=1}^{3} G_{i} \gamma_{i}^{2}}\right)
$$

s.t.

$$
\gamma_{i}^{2}\left(H_{i}+1\right) \leq 1, \quad i=1,2,3 .
$$

Note that the factor $1 / 2$ in $(6 a)$ is due to the equal duration of phases.

Moreover, we observe that (6) is a non-linear nonconvex optimization problem in the three variables $\left\{\gamma_{i}\right\}$.

\subsection{Decode-and-forward}

With DF a relay decodes the information received from $S$, which is transmitted coherently with the other relays towards $D$ [4]. A bottleneck of this scheme is the channel between $S$ and each relay, as the spectral efficiency is strongly limited by the worst channel $\min _{i}\left\{H_{i}\right\}$. For this reason, we also consider a selection of the relays involved in the operations. For each subset $\mathcal{S} \subseteq\{1,2,3\}$, in order to maximize the information rate from $S$ to $D$, we impose the equality of the spectral efficiency in both phases, i.e.,

$$
R^{(\mathrm{DF})}(\mathcal{S})=\lambda C\left(\min _{i \in \mathcal{S}}\left\{H_{i}\right\}\right)=(1-\lambda) C\left(\left(\sum_{i \in \mathcal{S}} \sqrt{G_{i}}\right)^{2}\right) .(7)
$$

The optimal value of $\lambda$ is obtained by solving (7) for each subset $\mathcal{S}$, i.e.,

$$
\lambda=\frac{C\left(\left(\sum_{i \in \mathcal{S}} \sqrt{G_{i}}\right)^{2}\right)}{C\left(\min _{i \in \mathcal{S}}\left\{H_{i}\right\}\right)+C\left(\left(\sum_{i \in \mathcal{S}} \sqrt{G_{i}}\right)^{2}\right)} .
$$

The network spectral efficiency is then computed optimizing the choice of $\mathcal{S}$, yielding

$$
R^{(\mathrm{DF})}=\max _{\mathcal{S} \leq\{1,2,3\}} R^{(\mathrm{DF})}(\mathcal{S})=\max _{\mathcal{S} \subseteq 1,2,3\}} \frac{C\left(\min _{i \in \mathcal{S}}\left\{H_{i}\right\}\right) C\left(\left(\sum_{i \in \mathcal{S}} \sqrt{G_{i}}\right)^{2}\right)}{C\left(\min _{i \in \mathcal{S}}\left\{H_{i}\right\}\right)+C\left(\left(\sum_{i \in \mathcal{S}} \sqrt{G_{i}}\right)^{2}\right)} .
$$

Note that (9) is an integer optimization problem, which can be easily solved by an exhaustive search among all the subsets of relay nodes.

\subsection{Broadcast-multiaccess}

Only for this section, we assume for simplicity that $H_{1} \geq$ $H_{2} \geq H_{3}$. In the BM scheme the first phase is a Gaussian broadcast channel [11], where $S$ transmits three messages $M_{1}, M_{2}$, and $M_{3}$ at rates $R_{1}, R_{2}$, and $R_{3}$, respectively, where $M_{1}$ is decoded only by relay $1, M_{2}$ is decoded by both relay 1 and relay 2 , and $M_{3}$ is decoded by all three relays. The second phase is a Gaussian multiple-access channel with correlated information [9], where relays send different but not independent information. We indicate with $\alpha_{1}, \alpha_{2}$, and $\alpha_{3}$ the powers used by $S$ to transmit the messages $M_{1}, M_{2}$, and $M_{3}$, respectively, $\gamma_{11}, \gamma_{12}$, and $\gamma_{1}$ the powers used by relay 1 to transmit $M_{1}, M_{2}$, and $M_{3}$, respectively, $\gamma_{21}$ and $\gamma_{2}$ the powers used by relay 2 to transmit $M_{2}$ and $M_{3}$, respectively, and $\gamma_{3}$ the power used by relay 3 to transmit $M_{3}$. Note that here we are extending the BM scheme with two relays of [8] to the case of three relays. Therefore, the network spectral efficiency is the solution of the following optimization problem:

$$
R^{(\mathrm{BM})}=\max _{\alpha_{i}, \gamma_{u}, \lambda \geq 0} R_{1}+R_{2}+R_{3}
$$

s.t.

$$
\begin{aligned}
& R_{1} \leq \lambda C\left(\alpha_{1} H_{1}\right) \\
& R_{2} \leq \lambda C\left(\frac{\alpha_{2} H_{2}}{1+\alpha_{1} H_{2}}\right) \\
& R_{3} \leq \lambda C\left(\frac{\alpha_{3} H_{3}}{1+\alpha_{1} H_{3}+\alpha_{2} H_{3}}\right) \\
& R_{1} \leq(1-\lambda) C\left(\gamma_{11} G_{1}\right) \\
& R_{1}+R_{2} \leq(1-\lambda) C\left(\gamma_{11} G_{1}+\left(\sqrt{\gamma_{12} G_{1}}+\sqrt{\gamma_{21} G_{2}}\right)^{2}\right), \\
& R_{1}+R_{2}+R_{3} \leq(1-\lambda) C\left(\gamma_{11} G_{1}+\left(\sqrt{\gamma_{12} G_{1}}+\sqrt{\gamma_{21} G_{2}}\right)^{2}+\right. \\
& \left.\quad\left(\sqrt{\gamma_{1} G_{1}}+\sqrt{\gamma_{2} G_{2}}+\sqrt{\gamma_{3} G_{3}}\right)^{2}\right)
\end{aligned}
$$




$$
\begin{aligned}
& \alpha_{1}+\alpha_{2}+\alpha_{3} \leq 1, \\
& \gamma_{11}+\gamma_{12}+\gamma_{1} \leq 1, \\
& \gamma_{21}+\gamma_{2} \leq 1, \\
& \gamma_{3} \leq 1, \\
& \lambda \leq 1 .
\end{aligned}
$$

Note that (i) constraints (10b)-(10d) represent the rate-region of the Gaussian broadcast channel between $S$ and the relays, (ii) constraints (10e)-(10g) represent the rate-region of the Gaussian multiple-access channel with correlated information between the relays and $D$, and (iii) constraints (10h)-(10k) represent the power constraints at node $S$ and at the relays, respectively. Similarly to (6), (10) is a non-linear non-convex optimization problem.

\section{Proposed schemes}

We propose now two schemes where the three relays are not forced to receive simultaneously at the same phase. For a scenario with three relays and two phases, we consider a configuration where two relays are transmitting to the destination, and the third is receiving from the source. We indicate with $R^{(A)}$ the spectral efficiency related to the couple of cooperative relays and with $R^{(B)}$ the spectral efficiency related to the remaining relay. Let $r_{\max }$ be the index of the relay with the best channel $H_{i}$, i.e., $r_{\max }=\arg \max _{i: \delta_{i}=0} H_{i}$, and let $r_{\min }$ be the index of the relay with the worst channel $H_{i}$, i.e., $r_{\min }=\arg \min _{i: \delta_{i}=0} H_{i}$.

\subsection{Adaptive BM scheme}

We first propose an extension of the BM scheme where the couple of cooperative relays operating synchronously employ Slepian-Wolf encoding to jointly transmit a common message at rate $R^{(C)}$ towards $D$, while relay $r_{\max }$ also transmits a private message at rate $R^{(P)}$, i.e., $R^{(A)}=R^{(P)}+R^{(C)}$. The resulting scheme is named adaptive BM (ABM). The private message is transmitted by $S$ with power $\alpha$ and by the selected relay with power $P_{r_{\max }}^{(P)}$. Furthermore, the third relay decodes an independent message received from $S$, which is then retransmitted towards $D$ at rate $R^{(B)}$. We recall that $\delta_{i}$ identifies the phase in which relay $i$ transmits. The achievable network spectral efficiency with this scheme can be written as

$$
R^{(\mathrm{ABM})}=\max _{\alpha, P_{r_{\max }^{(P)}}^{(P,}, \delta_{i}} R^{(P)}+R^{(C)}+R^{(B)}
$$

s.t.

$$
\begin{aligned}
& R^{(B)} \leq \lambda C\left(\sum_{i=1}^{3} G_{i} \delta_{i}\right) \\
& R^{(B)} \leq(1-\lambda) C\left(\sum_{i=1}^{3} H_{i} \delta_{i}\right), \\
& R^{(P)} \leq \lambda C\left(\alpha H_{r_{\max }}\right), \\
& R^{(C)} \leq \lambda C\left(\frac{(1-\alpha) H_{r_{\min }}}{1+\alpha H_{r_{\min }}}\right), \\
& R^{(P)} \leq(1-\lambda) C\left(P_{r_{\max }}^{(P)} G_{r_{\max }}\right), \\
& R^{(P)}+R^{(C)} \leq(1-\lambda) C\left(P_{r_{\max }^{(P)}} G_{r_{\max }}+\left(\sqrt{\left(1-P_{r_{\max }}^{(p)} G_{r_{\max }}\right.}+\sqrt{G_{r_{\min }}}\right)^{2}\right),
\end{aligned}
$$$$
\sum_{i=1}^{3} \delta_{i}=1, \quad \delta_{i} \in\{0,1\}, \quad i=1,2,3,
$$$$
r_{\max }=\arg \max _{i: \delta_{i}=0} H_{i}, \quad r_{\min }=\arg \min _{i: \delta_{i}=0} H_{i}
$$$$
0 \leq \lambda \leq 1, \quad 0 \leq \alpha \leq 1, \quad 0 \leq P_{r_{\max }}^{(P)} \leq 1
$$

The spectral efficiency of the non-cooperative relay (having index $i$, such that $\delta_{i}=1$ ) is suitably bounded in (11b) and (11c) for the two phases, respectively. For the cooperative relays (both having $\left.\delta_{i}=0\right)(11 \mathrm{~d}$ ) and (11e) bound the spectral efficiency in the first phase, while (11f) and (11g) give the bound for the common and private message in the second phase.

Note that (11) is a mixed integer programming problem.

\subsection{Adaptive BM with QAM quantization}

We now propose an alternative solution to Slepian-Wolf encoding used by cooperative relays in Section 3.1. In particular, we assume that the couple of cooperative relays employ QAM constellations, and we denote this scheme adaptive BM with QAM quantization (ABMQAM). In this scheme the central controller schedules a certain QAM symbol $a$ to be sent towards $D$. In the first phase, we assume link $H_{r_{\max }}$ is able to deliver all the information sent by $S$ to relay $r_{\max }$, which is then able to reconstruct the full symbol $a$. At the same time link $H_{r_{\text {min }}}$ is able to deliver only a quantized version to relay $r_{\text {min }}$, i.e., 


$$
a^{(q)}=a-a^{(e)},
$$

where $a^{(e)}$ is the quantization error. Hence relay $r_{\min }$ has a knowledge of $a^{(q)}$ only. By letting $b$ and $b^{(q)} \in\{0,2,4, \ldots$, $b$ be the sizes of the full QAM and the quantized QAM, respectively, assuming $\mathrm{E}\left[|a|^{2}\right]=1$ and following the quantized constellation design of [10], where $\mathrm{E}\left[a^{(e)}\right]=0$ and $\mathrm{E}$ $\left[a^{(q) *} a^{(e)}\right]=0$, we have

$$
f\left(b, b^{(q)}\right)=\mathrm{E}\left[\left|a^{(e)}\right|^{2}\right]=\frac{2^{b-b^{(q)}}-1}{2^{b}-1} .
$$

The signal transmitted by relay $r_{\text {min }}$ can be written as

$$
z_{r_{\min }}=\sqrt{P_{r_{\min }}^{(C)}} a^{(q)}
$$

while the signal transmitted by relay $r_{\max }$ is a suitable combination of $a^{(q)}$ and $a^{(e)}$, i.e.,

$$
z_{r_{\max }}=\sqrt{P_{r_{\max }}^{(C)}} a^{(q)}+\sqrt{P_{r_{\max }}^{(P)}} a^{(e)} .
$$

The powers $\left\{P_{r_{\max }}^{(C)}, P_{r_{\max }}^{(P)}, P_{r_{\min }}^{(C)}\right\}$ are chosen to reconstruct the full QAM symbol $a$ at node $D$ by imposing

$$
\sqrt{G_{r_{\max }} P_{r_{\max }}^{(C)}}+\sqrt{G_{r_{\min }} P_{r_{\min }}^{(C)}}=\sqrt{G_{r_{\max }} P_{r_{\max }^{(P)}}^{(P)}} .
$$

By using (16), the SNR at $D$ turns out to be $G_{r_{\max }} P_{r_{\max }}^{(P)}$ and we approximate the upper bound on the spectral efficiency from relays $r_{\max }$ and $r_{\min }$ to $D$ as

$$
R^{(A)} \leq(1-\lambda) \min \left\{b, C\left(G_{r_{\max }} P_{r_{\max }}^{(P)}\right)\right\},
$$

where we consider a maximum spectral efficiency $b$ related to the size of the constellation. Note that the symbol transmitted by relay $r_{\max }$ and the quantized QAM symbol transmitted by relay $r_{\min }$ combine at the destination into a QAM symbol that can be detected using conventional methods; indeed, node $D$ is unaware of the private transmission from relay $r_{\max }$, although it obtains the benefit of an higher SNR. With this transmission scheme the implementation complexity at node $D$ is significantly reduced with respect to the $A B M$ scheme because it does not need successive interference cancelation to decode both private and common messages.

The problem of finding the exact values of the spectral efficiencies of signals $a^{(q)}$ and $a^{(e)}$, which are sent through the Gaussian broadcast channel from $S$ to relays $r_{\max }$ and $r_{\min }$, respectively, is the problem of finding the spectral efficiencies of the messages after channel coding, interleaving, and constellation quantization. Even if entropy coding could be used to compute these quantities, its complexity may be too high for a practical system implementation. Hence we approximate the spectral efficiency of $a^{(q)}$ with $\min \left\{(1-\lambda) b^{(q)}, R^{(A)}\right\}$ and that of $a^{(e)}$ with $\min \left\{(1-\lambda)\left(b-b^{(q)}\right), R^{(A)}\right\}$. With this suboptimal method $S$ makes the best choice between transmitting the full stream of information bits (at rate $R^{(A)}$ ) or only the sequence of bits after quantization, at rate $(1-\lambda) b^{(q)}$ for $a^{(q)}$ and at rate $(1-\lambda)\left(b-b^{(q)}\right)$ for $a$ ${ }^{(e)}$. Denoting with $\mathscr{M}$ the set of available QAM constellations, the network spectral efficiency from $S$ to $D$ can be written as

$$
R^{(\mathrm{ABM}-\mathrm{QAM})}=\max _{\alpha, \lambda, \delta_{i}, P_{r_{\max }^{(P)}, P_{r_{\max }}^{(C)}, P_{\min }^{(C)}}^{(C)}} R^{(A)}+R^{(B)}
$$

s.t.

$$
\begin{aligned}
& R^{(B)} \leq \lambda C\left(\sum_{i=1}^{3} G_{i} \delta_{i}\right), \\
& R^{(B)} \leq(1-\lambda) C\left(\sum_{i=1}^{3} H_{i} \delta_{i}\right),
\end{aligned}
$$

$$
R^{(A)} \leq(1-\lambda) \min \left\{b, C\left(G_{r_{\max }} P_{r_{\max }}^{(P)}\right)\right\},
$$

$$
\left(1-f\left(b, b^{(q)}\right)\right) P_{r_{\max }^{(C)}}^{(C)} f\left(b, b^{(q)}\right) P_{r_{\max }^{(P)}}^{(P)},
$$

$$
\left(1-f\left(b, b^{(q)}\right)\right) P_{r_{\min }}^{(C)} \leq 1,
$$

$$
\begin{aligned}
& \sqrt{G_{r_{\max }} P_{r_{\max }}^{(C)}}+\sqrt{G_{r_{\min }} P_{r_{\min }}^{(C)}}=\sqrt{G_{r_{\max }} P_{r_{\max }}^{(P)}} \\
& \min \left\{(1-\lambda)\left(b-b^{(q)}\right), R^{(A)}\right\} \leq \lambda C\left(\alpha H_{r_{\max }}\right), \\
& \min \left\{(1-\lambda) b^{(q)}, R^{(A)}\right\} \leq \lambda C\left(\frac{(1-\alpha) H_{r_{\min }}}{1+\alpha H_{r_{\min }}}\right),
\end{aligned}
$$

$$
\begin{aligned}
& \sum_{i=1}^{3} \delta_{i}=1, \quad \delta_{i} \in\{0,1\}, \quad i=1,2,3, \\
& r_{\max }=\arg \max _{i: \delta_{i}=0} H_{i}, \quad r_{\min }=\arg \min _{i: \delta_{i}=0} H_{i}, \\
& 0 \leq \alpha \leq 1, \quad 0 \leq \lambda \leq 1, \\
& P_{r_{\max }}^{(C)} P_{r_{\max }}^{(P)}, P_{r_{\min }}^{(C)} \geq 0,
\end{aligned}
$$




$$
2^{b} \in \mathcal{M}, \quad b^{(q)} \in\{0,2,4, \ldots, b\} .
$$

Note that (18e) and (18f) represent the power constraints at relays $r_{\max }$ and $r_{\min }$, respectively. Similarly to (11), (18) is a mixed integer programming problem.

\section{Numerical results}

We consider that all nodes are located in a plane. Channel gains are related to the path loss, hence can be written as

$$
H_{i}=\frac{\kappa}{d_{S i}^{2}}, \quad G_{i}=\frac{\kappa}{d_{i D}^{2}},
$$

where $d_{S i}$ and $d_{i D}$ are the distances between $S$ and relay $i$ and between relay $i$ and $D$, respectively, and $\kappa$ is a normalization factor that determines the receive SNR for a unitary distance. We assume that source node $S$ is located at $\left(-d_{\mathrm{MAX}}, 0\right)$ and destination node $D$ at $\left(0, d_{\mathrm{MAX}}\right)$. For ABMQAM we consider the constellations used in 3GPP LTE [12], i.e., $\mathcal{M}=\{\mathrm{QPSK}, 16 \mathrm{QAM}, 64 \mathrm{QAM}\}$. Developed schemes are compared in terms of network spectral efficiency also considering the cut-set upper bound computed in Appendix 1.

Problems (6), (10), (11), and (18) belong to NP-hard class and we compute their solution by using standard global solver tools in GAMS [13], thanks to the limited size of the considered scenario.

Figure 2 shows the network spectral efficiency in terms of $-d_{\mathrm{MAX}}<d<d_{\mathrm{MAX}}$, by assuming that relay nodes move from $S$ to $D$ on a set of ellipses centered in $(0,0)$ with the semi-major axes long $d_{\mathrm{MAX}}$ and different values for the semi-minor axes. In detail, relay 1 is located at $\left(d, d_{\mathrm{MAX}} / 3 \sqrt{1-\left(d / d_{\mathrm{MAX}}\right)^{2}}\right)$, relay 2 at $(d, 0)$, and relay 3

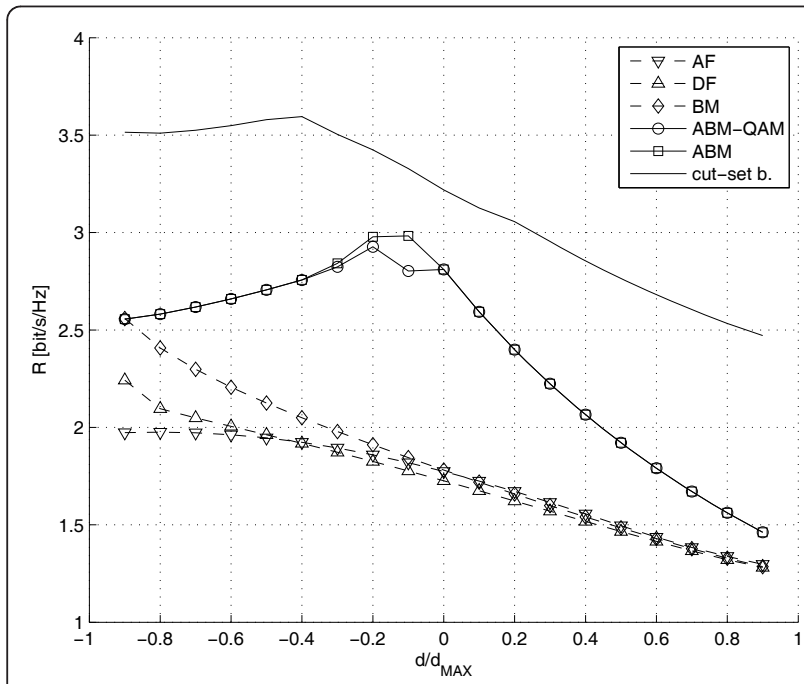

Figure 2 Network spectral efficiency in terms of $d$ for the network model described in Figure 3 assuming $\Gamma_{\mathrm{REF}}=2 \mathrm{~dB}$. at $\left(d_{1}-d_{\mathrm{MAX}} \sqrt{1-\left(d / d_{\mathrm{MAX}}\right)^{2}}\right)$ (see also Figure 3$)$ and we impose a reference signal to noise ratio at distance $2 d_{\mathrm{MAX}}$ as $\Gamma_{\mathrm{REF}}=\kappa /\left(2 d_{\mathrm{MAX}}\right)^{2}=2 \mathrm{~dB}$. We observe that schemes as $\mathrm{ABM}$ and $\mathrm{ABM}-\mathrm{QAM}$ significantly outperform the other schemes, which do not achieve spatial multiplexing, and ABM strictly outperforms ABM-QAM. As expected (see also [8]) BM strictly outperforms DF and DF outperforms AF when the relays are clustered around $S$, whereas AF strictly outperforms DF when the relays are half way between $S$ and $D$.

Figure 4 shows the average network spectral efficiency in terms of $\Gamma_{\text {REF }}$ by assuming that relay nodes are randomly dropped in a square centered in $(0,0)$ and whose sides have length $2\left(d_{\mathrm{Max}}-d_{\mathrm{MIN}}\right)$, where $d_{\mathrm{MIN}}$ is the minimum distance between $S$ or $D$ and each relay (see also Figure 5). We set $\kappa / d_{\text {MIN }}^{2}=30 \mathrm{~dB}$ which determines the maximum value assumed by $H_{i}$ and $G_{i}$. Note that with this value of $d_{\text {MIN }}$ the saturation of the spectral efficiency due to the use of a finite set of QAM constellations in ABM-QAM is negligible. As expected, the spectral efficiency achieved by all the schemes is an increasing function of $\Gamma_{\mathrm{REF}}$. Both $\mathrm{ABM}$ and $\mathrm{ABM}-\mathrm{QAM}$ still outperform DF and AF, while ABM strictly outperforms ABM-QAM. Moreover, the performance of $\mathrm{AF}$ and $\mathrm{DF}$ are now quite close.

We then consider a Rayleigh fading channel where each $\kappa$ variable in (19) is multiplied by an independent exponential random variable with unitary mean. Figure 6 shows the cumulative distribution function (CDF) of the spectral efficiency for the various schemes by imposing $\Gamma_{\mathrm{REF}}=2 \mathrm{~dB}$ and setting $d=0$ in the network of Figure 3. We observe that the spectral efficiency of the $\mathrm{ABM}$ schemes has a larger variance than that of $\mathrm{AF}, \mathrm{DF}$, and $\mathrm{BM}$ schemes as $\mathrm{ABM}$ are adaptive and thus are able to exploit the best channel conditions. We also observe that the average values of the spectral efficiencies are

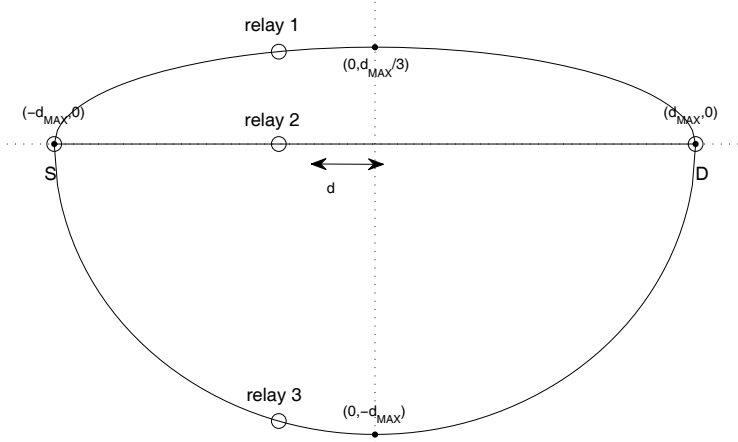

Figure 3 Network model considered in Figure 2 


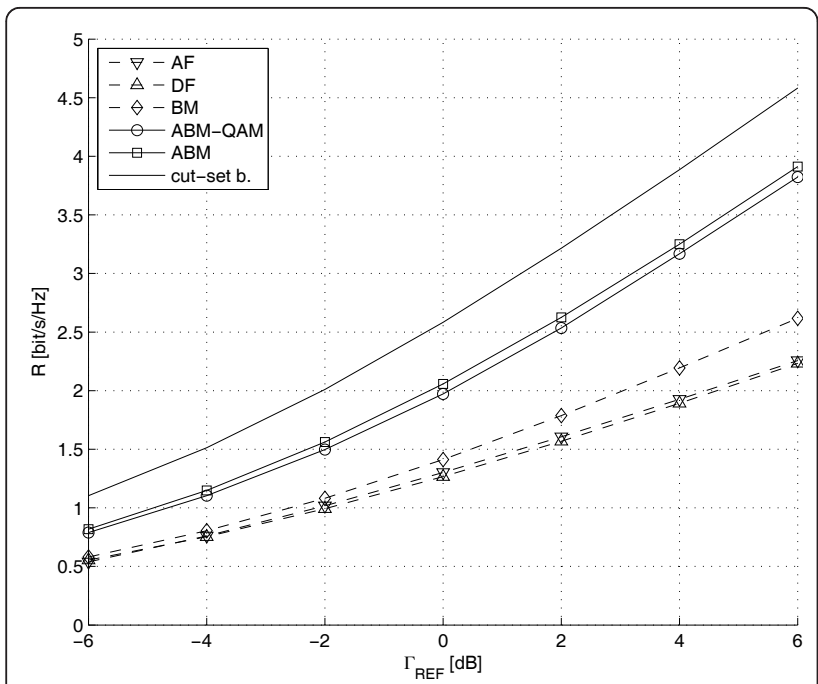

Figure 4 Average network spectral efficiency in terms of $\Gamma_{\mathrm{REF}}$ for the network model described in Figure 5

quite close to that obtained for non-fading channels with the same average SNR.

Note that the solutions of both ABM and ABM-QAM optimizations require a high computation time as most mixed integer programming problems. However, even if ABM strictly outperforms ABM-QAM, the implementation complexity of ABM-QAM is considerably lower as the destination does not require successive interference cancelation. Note that if we consider for example a cellular system, the complexity constraints are tighter at the mobile terminals with respect to base and relay stations.

\section{Conclusions}

In this article, we have considered a network with three half-duplex relays assisting the transmission of a source

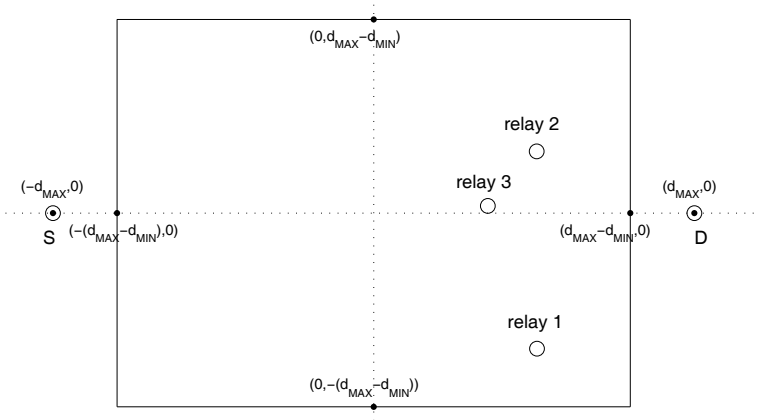

Figure 5 Network model considered in Figure 4

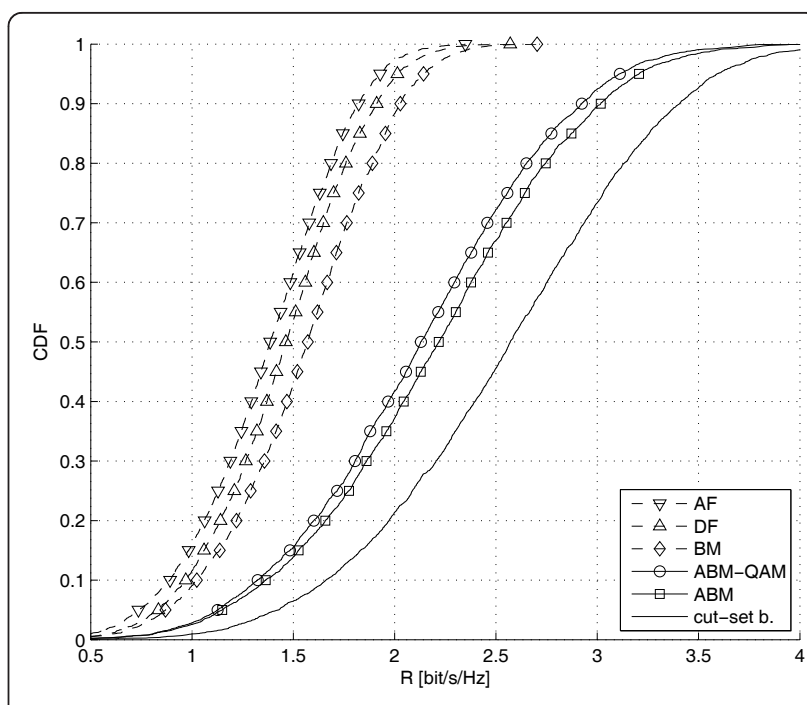

Figure $6 \mathrm{CDF}$ of the network spectral efficiency with $\Gamma_{\mathrm{REF}}=2$ $\mathrm{dB}$, relay 1 located at $\left(0, d_{\mathrm{MAX}} / 3\right)$, relay 2 at $(0,0)$, and relay 3 at $\left(0,-d_{\mathrm{MAX}}\right)$

towards a destination. Spatial multiplexing is achieved by allowing a single relay to transmit in odd phases and the other two relays to transmit in even phases. We have formalized the problem of maximizing network spectral efficiency when the cooperative relays employ (a) Slepian-Wolf encoding and (b) a suboptimal scheme based on QAM quantization. We have shown that in typical wireless scenarios ABM-QAM achieves the performance of $\mathrm{ABM}$ and outperforms other existing techniques such as DF and $\mathrm{AF}$.

\section{Appendix 1: cut-set upper bound}

Since $S$ and $D$ are always transmitting and receiving, respectively, there are at most $2^{3}=8$ configurations of $\left\{\delta_{i}\right\}$. Only for this appendix, we denote with $\bar{\lambda}$ the fraction of the total unitary time when no relay is transmitting, $\lambda$ when all relays are transmitting, $\lambda_{i}$ when only relay $i$ is transmitting and $\bar{\lambda}_{i}$ when only relay $i$ is not transmitting. Moreover, there are eight different "cuts" that separate $S$ from $D$, and each one is related to a constraint on the information-rate. The cut-set upper bound for the considered relay network can be expressed as [14]

$$
R^{\text {(cut - setb. })}=\max _{\left\{\bar{\lambda}, \lambda_{i}, \bar{\lambda}_{i}, \lambda\right\} \geq 0} R
$$

s.t.

$$
R \leq \bar{\lambda} C\left(\sum_{i} H_{i}\right)+\sum_{i} \lambda_{i} C\left(\sum_{j \neq i} H_{j}\right)+\sum_{i} \bar{\lambda}_{i} C\left(H_{i}\right),
$$




$$
\begin{aligned}
R \leq & \bar{\lambda} C\left(\sum_{j \neq i} H_{j}\right)+\lambda_{i}\left[C\left(\sum_{j \neq i} H_{j}\right)+C\left(G_{i}\right)\right]+\sum_{j \neq i} \lambda_{j} C\left(H_{k: k \neq i, j}\right)+ \\
& \sum_{j \neq i} \bar{\lambda}_{j}\left[C\left(H_{j}\right)+C\left(G_{i}\right)\right]+\lambda C\left(G_{i}\right), \quad i=1,2,3, \\
R \leq & \bar{\lambda} C\left(H_{i}\right)+\sum_{j \neq i} \lambda_{j}\left[C\left(H_{i}\right)+C\left(G_{j}\right)\right]+\bar{\lambda}_{i}\left[C\left(H_{i}\right)+C\left(\left(\sum_{j \neq i} \sqrt{G_{j}}\right)^{2}\right)\right]+ \\
& \sum_{j \neq i} \bar{\lambda}_{j} C\left(G_{k: k \neq i, j}\right)+\lambda C\left(\left(\sum_{j \neq i} \sqrt{G_{j}}\right)^{2}\right), \quad i=1,2,3, \\
R \leq & \sum_{i} \lambda_{i} C\left(G_{i}\right)+\sum_{i} \bar{\lambda}_{i} C\left(\left(\sum_{j \neq i} \sqrt{G_{j}}\right)^{2}\right)+\lambda C\left(\left(\sum_{i} \sqrt{G_{i}}\right)^{2}\right), \\
\bar{\lambda} & +\sum_{i}\left(\lambda_{i}+\bar{\lambda}_{i}\right)+\lambda \leq 1 .
\end{aligned}
$$

Note that (20) is a convex optimization problem.

\section{Competing interests}

The authors declare that they have no competing interests.

Received: 14 June 2011 Accepted: 29 March 2012

Published: 29 March 2012

\section{References}

1. TM Cover, A El Gamal, Capacity theorems for the relay channel. IEEE Trans Inf Theory. 25(5), 572-584 (1979). doi:10.1109/TIT.1979.1056084

2. A Sendonaris, E Erkip, B Aazhang, User cooperation diversity-part I and part II. IEEE Trans Commun. 51(11), 1927-1948 (2003). doi:10.1109/ TCOMM.2003.818096

3. A Høst-Madsen, J Zhang, Capacity bounds and power allocation for wireless relay channels. IEEE Trans Inf Theory. 51(6), 2020-2040 (2005), doi:10.1109/TIT.2005.847703

4. B Rankov, A Wittneben, Spectral efficient protocols for half-duplex fading relay channels. IEEE J Sel Areas Commun. 25(2), 379-389 (2007). doi:10.1109/JSAC.2007.070213

5. Y Yang, H Hu, J Xu, G Mao, Relay technologies for WiMax and LTEadvanced mobile systems. IEEE Commun Mag. 47(10), 100-105 (2009). doi:10.1109/MCOM.2009.5273815

6. A Reznik, SR Kulkarni, S Verdu, Degraded Gaussian multirelay channel: capacity and optimal power allocation. IEEE Trans Inf Theory. 50(12), 3037-3046 (2004). doi:10.1109/TIT.2004.838373

7. G Kramer, M Gastpar, P Gupta, Cooperative strategies and capacity theorems for relay networks. IEEE Trans Inf Theory. 51(9), 3037-3063 (2005). doi:10.1109/TIT.2005.853304

8. F Xue, S Sandhu, Cooperation in a half-duplex Gaussian diamond relay channel. IEEE Trans Inf Theory. 53(10), 3806-3814 (2007). doi:10.1109/ TIT.2007.904782

9. D Slepian, JK Wolf, A coding theorem for multiple access channels with correlated sources. Bell Syst Tech J. 52, 1037-1076 (1973)

10. P Baracca, S Tomasin, N Benvenuto, Downlink multicell processing employing QAM quantization under a constrained backhaul. in Proc IEEE Signal Processing Advances in Wireless Communications (SPAWC). 1, 1-5 (2011). doi:10.1109/SPAWC.2011.5990394

11. TM Cover, JA Thomas, Elements of Information Theory (John Wiley \& Sons, New York, 2006)

12. S Sesia, I Toufik, M Baker, LTE: The UMTS Long Term Evolution (John Wiley \& Sons, New York, 2009)

13. General algebraic modeling system (GAMS) http://www.gams.com

14. MA Khojastepour, A Sabharwal, B Aazhang, Bounds on achievable rates for general multi-terminal networks with practical constraints. in Proc Inf Process Sens Netw Second Int Work. 2634, 146-161 (2003) doi:10.1186/1687-1499-2012-128

Cite this article as: Baracca et al:: A practical scheduling and power/ constellation allocation for three relay networks. EURASIP Journal on Wireless Communications and Networking 2012 2012:128.

\section{Submit your manuscript to a SpringerOpen ${ }^{\mathcal{O}}$ journal and benefit from:}

- Convenient online submission

- Rigorous peer review

- Immediate publication on acceptance

- Open access: articles freely available online

- High visibility within the field

- Retaining the copyright to your article

Submit your next manuscript at $>$ springeropen.com 